\title{
The Factors related to Mothers' Intention to Vaccinate against Hepatitis A: Applying the Theory of Planned Behavior
}

\author{
Kyeong-Sook Cha ${ }^{1}$, Kyung Mi Kim ${ }^{2}$ \\ ${ }^{1}$ Associate Professor, Department of Nursing Science, Sun Moon University, Asan; ${ }^{2}$ Associate Professor, Department of Nursing Science, Chungbuk National \\ University, Cheonju, Korea
}

\begin{abstract}
Purpose: This study was conducted using the theory of planned behavior to analyze factors influencing mothers' intention to vaccinate their children against hepatitis A (HA). Methods: This descriptive study used a questionnaire. The participants were 100 mothers with children under 19 years. The collected data were analyzed by the t-test, analysis of variance, and the Mann-Whitney U test. The Pearson's correlation was used to test the correlations among variables. Stepwise multiple regression was used to identify factors related to HA vaccination intention. Results: The attitude of mothers with children under 19 to HA vaccination were quite positive (6.2 of 7), and their perceived behavioral control (5.73 of 7), subjective norm (5.54 of 7), and vaccination intention (5.96 of 7) were relatively high. Attitude toward HA vaccination was the strongest influencing factor, followed by subjective norm. The explanatory power of attitude and subjective norm for HA vaccination was $84 \%$. Conclusion: HA vaccination is very important for preventing HA. Mothers' positive attitudes toward HA vaccination were an important factor influencing their children's HA vaccination. Providing education on the benefits of HA vaccination and the vaccination schedule may foster positive attitude toward vaccination.
\end{abstract}

Key words: Hepatitis; Vaccination; Intention; Behavior

\section{Corresponding author Kyung Mi Kim \\ https://orcid.org/0000-0001-9799-0677}

Department of Nursing Science, Chungbuk National University, 1 Chungdae-ro, Seowon-gu, Cheonju 28644, Korea

TEL +82-43-249-1783 FAX +82-43-266-1710

E-MAIL icpkim@chungbuk.ac.kr

Received Jul 5, 2018 Revised Aug 31, 2018 Accepted Sep 24, 2018
(-) This is an Open Access article distributed under the terms of the Creative Commons Attribution NonCommercial License (http://creativecommons.org/licenses/by-nc/4.0/) which permits unrestricted noncommercial use, distribution, and reproduction in any medium, provided the original work is properly cited.

\section{INTRODUCTION}

Hepatitis A (HA) affects health of infants and adolescents in underdeveloped and developing countries and affects the health of adults in more economically developed countries. It is a highly contagious disease transmitted through water or food contaminated by the stools of carriers [1,2]. HA rarely has complications, never becomes chronic, and its prognosis is relatively good. However, recently, some atypical clinical aspects have emerged, such as persistent hepatitis and recurrent hepatitis, and in some severe cases, death has occurred due to fulminating liver failure [3].

The HA antibody prevalence rate varies according to the economic level and hygiene of a particular country. In the United States, the HA seroprevalence rate was 31.3\% overall, $9.4 \%$ for ages $6 \sim 11$, and $74.6 \%$ over the age of 70 [4]. Similarly, the HA seroprevalence rate in Korea is low among children and young adults, but high among adults over 40 [5]. In South Korea in the 1980s, more than $90 \%$ of adults were immune to HA through natural infection [6]. However, the most recently reported seropositivity rate among people between 15 and 29 was between $11.7 \%$ and $13.0 \%$ [7]. In Korea, with improvements in economic prosperity and hygiene, fewer people have 
acquired natural immunity [8]. Cases of HA contracted overseas have also increased, as overseas travel to HA-endemic areas has become more common [8]. Accordingly, the risk of acute HA has become higher in children and young adults. Since 2008, the number of HA cases has increased rapidly, earning it the designation of a notifiable disease in 2011 [1].

Vaccination and practicing enhanced hygiene are steps recommended to prevent HA [1]. However, HA vaccination was not included in the mandatory vaccination list in Korea as of 2015, making vaccination against HA a personal choice. Oh and Park [9], in their study of university students, reported that age was a factor that influenced HA vaccination. Choi et al. [10] reported that decisions to vaccinate were made by parents, particularly by mothers, and Bardenheier et al. [11] reported that insufficient parental awareness of HA vaccination affected the vaccination rate.

Ajzen [12], who examined the factors that decisively influence human behaviors, considered "intention for behavior" to be an important variable in predicting behaviors. The theory of planned behavior (TPB) consists of 4 key concepts: attitude toward the behavior, subjective norm, perceived behavioral control, and intention. TPB has been used in the fields of sociopsychology $[13,14]$ and healthcare to identify predictors and influencing factors [15,16]. Through a meta-analysis, Armitage and Conner [17] suggested that TPB is effective in predicting intention and behaviors. Researchers can also use TPB to explain vaccination intentions. TPB has been mainly applied to studies explaining and predicting cervical-cancer prevention behaviors related to the human papilloma virus vaccine [1820]. Because the incidence of HA in Korea is relatively high among members of younger generations, active efforts are needed to improve the vaccination rate. Therefore, understanding the attitude toward the behavior, subjective norm, and perceived behavioral control as factors that influence mothers' intention to have their children receive HA vaccination may contribute to an increase in vaccination rates. In Korea, several studies [21,22] were conducted on factors related to HA vaccination in university students. A study on knowledge and health beliefs regarding HA vaccination [23] was conducted among parents. However, few studies have directly described the application of TPB for HA vaccination. Accordingly, this study aimed to contribute to the development of efficient nursing interventions designed to increase the HA vaccination rate and to prevent infection by using TPB to analyze factors influencing the HA vaccination intention of mothers with children under 19.

\section{METHODS}

\section{Design and Sampling}

This study was conducted as a descriptive survey, performed following approval from the university Institutional Review Board (***-2014-08-002). Participants were mothers with children under 19 who lived in Seoul and Gyeonggi Province. Participants were selected through convenience sampling. The researcher visited community organizations, churches, and school-parent meetings from June to August 2015 and explained the purpose of the study to candidates. All participants provided informed consent, and then answered the questionnaires. Participants were informed that participation was voluntary. Each participant took approximately 10 minutes to complete the survey. The sample size was determined using the G-Power 3.1 program. The minimum number of participants for the required sample size was 99 for an effect size of .15, a significance level of .05 , a test power .90, and three independent variables in multiple regression. To account for discarded responses (approximately 10\%) and to meet the minimum sample size, questionnaires were distributed to 109 participants; 100 questionnaires were analyzed as valid data, with 9 questionnaires excluded due to inadequate answers.

\section{Study Tool}

This study used a structured questionnaire consisting of questions about participants' general characteristics, hepatitisrelated characteristics, and TPB-related variables (attitude toward HA vaccination, perceived behavioral control, subjective norm, and vaccination intention). The questions on attitude toward HA vaccination and TPB-related variables were based on previous studies [19]. Two nursing professors and 1 infection-control nurse reviewed the questions for content validity.

\section{1) General characteristics and hepatitis-related characteristics}

Questions on general characteristics included age, religion, education, employment, number of children, and economic level. Five questions on hepatitis-related characteristics asked whether participants had heard of HA, whether they had received education about HA, their awareness of HA vaccination, whether they had been vaccinated against $\mathrm{HA}$, and their history of HA. Three questions were about hepatitis, and asked whether they are infected with or carriers of hepatitis B and hepatitis $\mathrm{C}$, and whether they have hepatitis-infected individuals or carriers among their family members; whether they had vaccinated their children against HA; and whether they planned to vaccinate their children.

\section{2) Attitude toward hepatitis A vaccination}

Attitude is defined as the level of positive or negative eval- 
uation of an individual's performance of a certain behavior [23]. In the present study, attitude referred to participants' positive or negative perceptions about HA vaccination. Questions consisted of 3 items measured on a 7-point scale, from 1 (absolutely not) to 7 (absolutely yes). A higher score indicated a more positive attitude toward HA vaccination. The Cronbach's $\alpha$ of this tool was .97 .

\section{3) Perceived behavioral control}

Perceived behavioral control is defined as the perception of the ease or difficulty of an individual's particular behavior [4]. In the present study, perceived behavioral control meant how difficult or easy participants perceived obtaining an HA vaccination to be. The questions consisted of 5 items measured on a 7-point scale, from 1 (absolutely not) to 7 (absolutely yes). Higher scores indicated that obtaining an $\mathrm{HA}$ vaccination was perceived as relatively easy. The Cronbach's $\alpha$ of this tool was .76.

\section{4) Subjective norm}

The concept of subjective norm denotes the social pressure that an individual subjectively perceives about the performance of a certain behavior [23]. In the present study, subjective norm indicated the degree of pressure the participant recognized from significant people in their lives with regard to HA vaccination. This section comprised 3 questions measured on a 7-point scale, from 1 (absolutely not) to 7 (absolutely yes). Higher scores indicated a greater subjective norm against HA vaccination; in other words, participants experienced greater pressure from those around them to vaccinate. The Cronbach's $\alpha$ of this tool was .94.

\section{5) Vaccination intention}

Intention is an individual's level of willingness to perform a certain behavior [23]. In the present study, vaccination intention meant the degree to which the participant would voluntarily make an effort to have her children receive the HA vaccination. This section of the questionnaire comprised 3 questions measured on a 7-point scale, from 1 (absolutely not) to 7 (absolutely yes). Higher scores indicated greater HA vaccination intention. The Cronbach's $\alpha$ of this tool was .98 .

\section{Data Analysis}

The collected data were analyzed with SPSS version 22.0 (IBM Corp., Armonk, NY, USA). The frequency, percentage, mean, and standard deviation were used to describe participants' general characteristics and hepatitis-related characteristics. The t-test, analysis of variance, and the Mann-Whitney $U$ test were used to analyze differences in attitude toward HA vaccination, perceived behavioral control, subjective norm, and vaccination intention aligned with participants' general and hepatitis-related characteristics. The Scheffé test was conducted as a post-comparison test. Correlations among attitude toward HA vaccination, perceived behavioral control, subjective norm, and vaccination intention were assessed by the Pearson's correlation. Stepwise multiple regression was used to identify influencing factors related to HA vaccination intention.

\section{RESULTS}

\section{Attitude toward HA Vaccination, Perceived Behavio- ral Control, Subjective Norm, and Vaccination Inten- tion according to Participants' General Character- istics}

The mean score for attitude toward HA vaccination was 6.20 of 7 ; for perceived behavioral control, 5.73 of 7; for subjective norm, 5.54 of 7; and for vaccination intention, 5.96 of 7 .

Those who were religious (5.87) had higher perceived behavioral control than those who were not (5.46) $(\mathrm{t}=2.43, p=$ .017). Attitude toward HA vaccination ( $\mathrm{F}=7.30, p=.001)$, perceived behavioral control $(\mathrm{F}=3.68, p=.029)$, subjective norm $(\mathrm{F}=4.15, p=.019)$, and HA vaccination intention $(\mathrm{F}=5.71, p=$ .004) were significantly different according to level of education, with college graduates scoring higher than those who had graduated from graduate school. For perceived behavioral control, high school graduates (5.81) and college graduates (5.80) showed higher scores than those who had graduated from graduate school (5.15) (Table 1).

\section{Attitude toward HA Vaccination, Perceived Behavioral Control, Subjective Norm, and Vaccination Intention according to Participants' HA-related Characteristics}

Among the 100 participants, $89 \%$ had heard of $\mathrm{HA}$ and 22\% had received education about HA, whereas $79 \%$ had heard of HA vaccination, $35 \%$ had received the vaccine, $7 \%$ had hepatitis carriers among their family members, and $59 \%$ had had their children receive an HA vaccination. Eighty-four percent of participants responded they planned to vaccinate their children $(t=8.56, p<.001)$.

The level of positive attitude to HA vaccination was significantly higher among those who had been vaccinated against HA $(t=2.88, p=.005)$, those who had had their children vaccinated against $\mathrm{HA}(\mathrm{F}=12.47, p<.001)$, and those who planned to have their children receive HA vaccination $(t=8.56, p<$ .001). The perceived behavioral control score was significantly higher among those who had received HA education $(\mathrm{F}=7.29$, 
Table 1. Differences in Dependent Variables according to Descriptive Characteristics

$(N=100)$

\begin{tabular}{|c|c|c|c|c|c|c|c|c|c|c|}
\hline \multirow[b]{2}{*}{ Characteristic } & \multirow[b]{2}{*}{ Categories } & \multirow{2}{*}{$\begin{array}{l}\mathrm{n}(\%) \text { or } \\
\mathrm{M} \pm \mathrm{SD}\end{array}$} & \multicolumn{2}{|c|}{ Attitude } & \multicolumn{2}{|c|}{ Control } & \multicolumn{2}{|c|}{ Norm } & \multicolumn{2}{|c|}{ Intention } \\
\hline & & & $\mathrm{M} \pm \mathrm{SD}$ & $\begin{array}{c}\mathrm{t} \text { or } \mathrm{F} \\
(p)\end{array}$ & $\mathrm{M} \pm \mathrm{SD}$ & $\begin{array}{c}\text { t or F } \\
(p)\end{array}$ & $\mathrm{M} \pm \mathrm{SD}$ & $\begin{array}{l}\mathrm{t} \text { or } \mathrm{F} \\
(p)\end{array}$ & $\mathrm{M} \pm \mathrm{SD}$ & $\begin{array}{c}\text { t or F } \\
(p)\end{array}$ \\
\hline Age (year) & & $39.88 \pm 5.94$ & & & & & & & & \\
\hline Religion & $\begin{array}{l}\text { No } \\
\text { Yes }\end{array}$ & $\begin{array}{l}35(35.0) \\
65(65.0)\end{array}$ & $\begin{array}{l}6.12 \pm 1.30 \\
6.24 \pm 1.11\end{array}$ & $\begin{array}{c}0.47 \\
(.638)\end{array}$ & $\begin{array}{l}5.46 \pm 0.86 \\
5.87 \pm 0.75\end{array}$ & $\begin{array}{c}2.43 \\
(.017)\end{array}$ & $\begin{array}{l}5.78 \pm 1.21 \\
5.41 \pm 1.31\end{array}$ & $\begin{array}{c}1.38 \\
(.170)\end{array}$ & $\begin{array}{l}5.88 \pm 1.47 \\
6.00 \pm 1.20\end{array}$ & $\begin{array}{c}0.43 \\
(.664)\end{array}$ \\
\hline Education & $\begin{array}{l}\text { High school }^{\mathrm{a}} \\
\text { College }^{\mathrm{b}} \\
\text { Graduate school }^{\mathrm{c}}\end{array}$ & $\begin{array}{l}21(21.0) \\
67(67.0) \\
12(12.0)\end{array}$ & $\begin{array}{l}5.90 \pm 1.36 \\
6.46 \pm 0.81 \\
5.22 \pm 1.87\end{array}$ & $\begin{array}{l}7.30 \\
(.001) \\
\mathrm{b}>\mathrm{c}^{*}\end{array}$ & $\begin{array}{l}5.81 \pm 0.66 \\
5.80 \pm 0.74 \\
5.15 \pm 1.19\end{array}$ & $\begin{array}{c}3.68 \\
(.029) \\
a, b>c^{*}\end{array}$ & $\begin{array}{l}5.14 \pm 1.40 \\
5.78 \pm 1.13 \\
4.86 \pm 1.52\end{array}$ & $\begin{array}{l}4.15 \\
(.019) \\
\mathrm{b}>\mathrm{c}^{*}\end{array}$ & $\begin{array}{l}5.74 \pm 1.34 \\
6.21 \pm 1.05 \\
4.94 \pm 1.90\end{array}$ & $\begin{array}{l}5.71 \\
(.004) \\
b>c^{*}\end{array}$ \\
\hline Employment & $\begin{array}{l}\text { No } \\
\text { Yes }\end{array}$ & $\begin{array}{l}29(29.0) \\
71(71.0)\end{array}$ & $\begin{array}{l}6.04 \pm 1.16 \\
6.22 \pm 1.24\end{array}$ & $\begin{array}{c}0.66 \\
(.507)\end{array}$ & $\begin{array}{l}5.86 \pm 0.69 \\
5.68 \pm 0.88\end{array}$ & $\begin{array}{c}0.99 \\
(.321)\end{array}$ & $\begin{array}{l}5.48 \pm 1.37 \\
5.54 \pm 1.27\end{array}$ & $\begin{array}{c}0.21 \\
(.829)\end{array}$ & $\begin{array}{l}6.01 \pm 1.09 \\
5.92 \pm 1.40\end{array}$ & $\begin{array}{c}0.27 \\
(.781)\end{array}$ \\
\hline $\begin{array}{l}\text { Number of } \\
\text { children }\end{array}$ & $\begin{array}{l}1 \\
2 \sim 3\end{array}$ & $\begin{array}{l}52(52.0) \\
48(48.0)\end{array}$ & $\begin{array}{l}6.13 \pm 1.18 \\
6.28 \pm 1.16\end{array}$ & $\begin{array}{c}0.66 \\
(.508)\end{array}$ & $\begin{array}{l}5.68 \pm 0.78 \\
5.83 \pm 0.79\end{array}$ & $\begin{array}{c}0.91 \\
(.359)\end{array}$ & $\begin{array}{l}5.41 \pm 1.19 \\
5.65 \pm 1.36\end{array}$ & $\begin{array}{c}0.92 \\
(.358)\end{array}$ & $\begin{array}{l}5.83 \pm 1.37 \\
6.10 \pm 1.19\end{array}$ & $\begin{array}{c}1.00 \\
(.318)\end{array}$ \\
\hline $\begin{array}{l}\text { Monthly income } \\
\text { (10,000 won) }\end{array}$ & $\begin{array}{l}\text { Below } 300 \\
300 \sim 500 \\
\text { Above } 500\end{array}$ & $\begin{array}{c}486.00 \pm 175.26 \\
11(11.0) \\
31(31.0) \\
58(58.0)\end{array}$ & $\begin{array}{l}6.21 \pm 1.10 \\
6.39 \pm 0.78 \\
6.09 \pm 1.36\end{array}$ & $\begin{array}{c}0.67 \\
(.512)\end{array}$ & $\begin{array}{l}5.81 \pm 0.96 \\
5.80 \pm 0.76 \\
5.67 \pm 0.81\end{array}$ & $\begin{array}{c}0.32 \\
(.723)\end{array}$ & $\begin{array}{l}5.48 \pm 1.25 \\
5.66 \pm 1.20 \\
5.48 \pm 1.34\end{array}$ & $\begin{array}{c}0.21 \\
(.807)\end{array}$ & $\begin{array}{l}6.24 \pm 1.05 \\
6.11 \pm 1.05 \\
5.82 \pm 1.45\end{array}$ & $\begin{array}{c}0.78 \\
(.458)\end{array}$ \\
\hline Total & & $100(100.0)$ & $6.20 \pm 1.18$ & & $5.73 \pm 0.81$ & & $5.54 \pm 1.28$ & & $5.96 \pm 1.30$ & \\
\hline
\end{tabular}

*Scheffé test, $p<.050$.

$p=.001)$, those who had contracted HA at some point $(\mathrm{U}=9.50$, $p=.009)$, and those who planned to have their children receive HA vaccination $(t=2.01, p=.047)$. The mean score for subjective norm was higher among those who had had their children receive $\mathrm{HA}$ vaccination $(\mathrm{F}=6.21, p=.003)$ and those who planned to do so $(\mathrm{t}=3.67, p<.001)$. The mean score for HA vaccination intention was significantly higher among those who had been vaccinated against HA ( $t=2.47, p=.015)$, those who planned for their children to receive HA vaccination $(t=8.59$, $p<.001$ ), and those who had had their children receive HA vaccination $(\mathrm{F}=14.37, p<.001)$ (Table 2$)$.

\section{Correlations among Attitude toward HA Vaccination, Perceived Behavioral Control, Subjective Norm, and Vaccination Intention}

Participants' intention of HA vaccination was significantly correlated with their attitude toward HA vaccination $(\mathrm{r}=.91$, $p<.001)$, perceived behavioral control $(\mathrm{r}=.58, p<.001)$, and subjective norm $(\mathrm{r}=.56, p<.001)$ (Table 3$)$.

\section{Factors Influencing HA Vaccination Intention}

Multicollinearity, tolerance, and the variance inflation factor (VIF) were performed to satisfy the assumptions of multiple regression analysis. The correlation coefficient among the independent variables was .09 .70. No variable had an explanatory power over .80 . Tolerance was $.51 \sim .84$, and the VIF was 1.18 1.94, which did not exceed the standard [13]. Therefore, multicollinearity was not present.

When performing multiple regression analysis by inputting variables stepwise, the regression model was significant $(\mathrm{F}=252.35, p<.001)$. Two variables were significant in influencing participants' HA vaccination intention: attitude toward HA vaccination $(\beta=.85)$ was the strongest influencing factor, followed by subjective norm $(\beta=.10)$. This outcome indicated that HA vaccination intention was higher when mothers had a positive attitude toward it and when the subjective norm score was higher. The explained variance for HA vaccination intention was $84 \%$ (Table 4 ).

\section{DISCUSSION}

When a large population is vulnerable to HA infection, as in Korea, a sudden epidemic can be caused by something as simple as contaminated food. The most cost-effective strategy for reducing HA infection in a short time is to increase herd immunity through vaccination [24]. In Korea, HA vaccination started in 1997, and complimentary vaccination has been provided to children under 12 years of age since May 2015 [21,25]. However, those over 12 years old pay approximately 160,000 won (approximately US\$140) for the 2-step vaccination process in Korea [26]. Therefore, youths between 13 and 19 who depend on their parents financially cannot make their own choices about HA vaccination. Because young people's attitude toward vaccination are influenced by their mothers' atti- 
Table 2. Differences in Dependent Variables according to Hepatitis-related Characteristics

$(N=100)$

\begin{tabular}{|c|c|c|c|c|c|c|c|c|c|c|}
\hline \multirow[b]{2}{*}{ Variables } & \multirow[b]{2}{*}{ Categories } & \multirow[b]{2}{*}{$\mathrm{n}(\%)$} & \multicolumn{2}{|c|}{ Attitude } & \multicolumn{2}{|c|}{ Control } & \multicolumn{2}{|c|}{ Norm } & \multicolumn{2}{|c|}{ Intention } \\
\hline & & & $\mathrm{M} \pm \mathrm{SD}$ & $\begin{array}{l}\mathrm{t} \text { or } \mathrm{F} \text { or } \\
\mathrm{U}(p)\end{array}$ & $\mathrm{M} \pm \mathrm{SD}$ & $\begin{array}{l}\mathrm{t} \text { or } \mathrm{F} \text { or } \\
\mathrm{U}(p)\end{array}$ & $\mathrm{M} \pm \mathrm{SD}$ & $\begin{array}{l}\mathrm{t} \text { or } \mathrm{F} \text { or } \\
\mathrm{U}(p)\end{array}$ & $\mathrm{M} \pm \mathrm{SD}$ & $\begin{array}{c}\text { t or F or } \\
U(p)\end{array}$ \\
\hline $\begin{array}{l}\text { Have you ever heard } \\
\text { about hepatitis A? }\end{array}$ & $\begin{array}{l}\text { No } \\
\text { Yes }\end{array}$ & $\begin{array}{l}11(11.0) \\
89(89.0)\end{array}$ & $\begin{array}{l}5.72 \pm 1.42 \\
6.25 \pm 1.14\end{array}$ & $\begin{array}{c}1.41 \\
(.161)\end{array}$ & $\begin{array}{l}5.58 \pm 0.83 \\
5.75 \pm 0.81\end{array}$ & $\begin{array}{c}0.64 \\
(.519)\end{array}$ & $\begin{array}{l}5.27 \pm 1.27 \\
5.57 \pm 1.29\end{array}$ & $\begin{array}{c}0.72 \\
(.467)\end{array}$ & $\begin{array}{l}5.39 \pm 1.22 \\
6.03 \pm 1.29\end{array}$ & $\begin{array}{c}1.55 \\
(.124)\end{array}$ \\
\hline $\begin{array}{l}\text { Have you ever been } \\
\text { educated about } \\
\text { hepatitis A? }\end{array}$ & $\begin{array}{l}\mathrm{No}^{\mathrm{a}} \\
\mathrm{Yes}^{\mathrm{b}} \\
\text { Not know }^{\mathrm{c}}\end{array}$ & $\begin{array}{c}70(70.0) \\
22(22.0) \\
8(8.0)\end{array}$ & $\begin{array}{l}6.09 \pm 1.24 \\
6.63 \pm 0.85 \\
5.91 \pm 1.20\end{array}$ & $\begin{array}{c}2.04 \\
(.135)\end{array}$ & $\begin{array}{l}5.61 \pm 0.79 \\
6.26 \pm 0.51 \\
5.32 \pm 1.01\end{array}$ & $\begin{array}{c}7.29 \\
(.001) \\
a, c<b^{*}\end{array}$ & $\begin{array}{l}5.52 \pm 1.19 \\
5.81 \pm 1.53 \\
4.87 \pm 1.24\end{array}$ & $\begin{array}{l}1.60 \\
(.205)\end{array}$ & $\begin{array}{l}5.84 \pm 1.30 \\
6.42 \pm 1.16 \\
5.75 \pm 1.46\end{array}$ & $\begin{array}{c}1.82 \\
(.167)\end{array}$ \\
\hline $\begin{array}{l}\text { Have you ever heard of } \\
\text { the hepatitis A vaccine? }\end{array}$ & $\begin{array}{l}\text { No } \\
\text { Yes }\end{array}$ & $\begin{array}{l}21(21.0) \\
79(79.0)\end{array}$ & $\begin{array}{l}5.93 \pm 1.13 \\
6.27 \pm 1.19\end{array}$ & $\begin{array}{l}1.15 \\
(.252)\end{array}$ & $\begin{array}{l}5.64 \pm 0.76 \\
5.75 \pm 0.82\end{array}$ & $\begin{array}{c}0.53 \\
(.595)\end{array}$ & $\begin{array}{l}5.53 \pm 1.26 \\
5.54 \pm 1.29\end{array}$ & $\begin{array}{c}0.01 \\
(.999)\end{array}$ & $\begin{array}{l}5.76 \pm 1.20 \\
6.01 \pm 1.32\end{array}$ & $\begin{array}{c}0.79 \\
(.427)\end{array}$ \\
\hline $\begin{array}{l}\text { Have you ever been } \\
\text { vaccinated for hepatitis } \\
\text { A? }\end{array}$ & $\begin{array}{l}\text { No } \\
\text { Yes }\end{array}$ & $\begin{array}{l}65(65.9) \\
35(35.0)\end{array}$ & $\begin{array}{l}5.95 \pm 1.32 \\
6.64 \pm 0.65\end{array}$ & $\begin{array}{c}2.88 \\
(.005)\end{array}$ & $\begin{array}{l}5.66 \pm 0.83 \\
5.85 \pm 0.76\end{array}$ & $\begin{array}{c}1.13 \\
(.261)\end{array}$ & $\begin{array}{l}5.45 \pm 1.33 \\
5.70 \pm 1.18\end{array}$ & $\begin{array}{c}0.94 \\
(.349)\end{array}$ & $\begin{array}{l}5.73 \pm 1.45 \\
6.39 \pm 0.80\end{array}$ & $\begin{array}{c}2.47 \\
(.015)\end{array}$ \\
\hline $\begin{array}{l}\text { Have you ever suffered } \\
\text { from hepatitis A? }\end{array}$ & $\begin{array}{l}\text { No } \\
\text { Yes }\end{array}$ & $\begin{array}{c}98(98.0) \\
2(2.0)\end{array}$ & $\begin{array}{l}6.18 \pm 1.18 \\
7.00 \pm 0.00\end{array}$ & $\begin{array}{l}53.00 \\
(.300)\end{array}$ & $\begin{array}{l}5.70 \pm 0.80 \\
6.90 \pm 0.14\end{array}$ & $\begin{array}{c}9.50 \\
(.009)\end{array}$ & $\begin{array}{l}5.51 \pm 1.28 \\
6.83 \pm 0.23\end{array}$ & $\begin{array}{l}37.00 \\
(.076)\end{array}$ & $\begin{array}{l}5.94 \pm 1.30 \\
7.00 \pm 0.00\end{array}$ & $\begin{array}{l}39.00 \\
(.166)\end{array}$ \\
\hline $\begin{array}{l}\text { Are you currently } \\
\text { infected with or a } \\
\text { carrier of hepatitis B? }\end{array}$ & $\begin{array}{l}\text { No } \\
\text { Yes }\end{array}$ & $\begin{array}{c}93(93.0) \\
7(7.0)\end{array}$ & $\begin{array}{l}6.16 \pm 1.21 \\
6.71 \pm 0.48\end{array}$ & $\begin{array}{c}1.19 \\
(.234)\end{array}$ & $\begin{array}{l}5.74 \pm 0.79 \\
5.54 \pm 1.04\end{array}$ & $\begin{array}{c}0.63 \\
(.526)\end{array}$ & $\begin{array}{l}5.53 \pm 1.30 \\
5.57 \pm 1.04\end{array}$ & $\begin{array}{c}0.06 \\
(.947)\end{array}$ & $\begin{array}{l}5.93 \pm 1.31 \\
6.28 \pm 1.07\end{array}$ & $\begin{array}{c}0.67 \\
(.499)\end{array}$ \\
\hline $\begin{array}{l}\text { Are you currently } \\
\text { infected with or a } \\
\text { carrier of hepatitis C? }\end{array}$ & $\begin{array}{l}\text { No } \\
\text { Yes }\end{array}$ & $\begin{array}{c}99(99.0) \\
1(1.0)\end{array}$ & $\begin{array}{l}6.19 \pm 1.18 \\
7.00 \pm 0.00\end{array}$ & $\begin{array}{l}27.00 \\
(.550)\end{array}$ & $\begin{array}{l}5.72 \pm 0.81 \\
6.00 \pm 0.00\end{array}$ & $\begin{array}{l}34.50 \\
(.400)\end{array}$ & $\begin{array}{l}5.54 \pm 1.29 \\
5.00 \pm 0.00\end{array}$ & $\begin{array}{l}31.00 \\
(.340)\end{array}$ & $\begin{array}{l}5.95 \pm 1.30 \\
6.33 \pm 0.00\end{array}$ & $\begin{array}{l}49.00 \\
(.520)\end{array}$ \\
\hline $\begin{array}{l}\text { Do you have a hepatitis- } \\
\text { infected individual or } \\
\text { carrier among your } \\
\text { family members? }\end{array}$ & $\begin{array}{l}\text { No } \\
\text { Yes }\end{array}$ & $\begin{array}{c}93(93.0) \\
7(7.0)\end{array}$ & $\begin{array}{l}6.21 \pm 1.17 \\
5.95 \pm 1.28\end{array}$ & $\begin{array}{c}0.57 \\
(.568)\end{array}$ & $\begin{array}{l}5.73 \pm 0.83 \\
5.65 \pm 0.45\end{array}$ & $\begin{array}{c}0.25 \\
(.802)\end{array}$ & $\begin{array}{l}5.51 \pm 1.28 \\
5.85 \pm 1.34\end{array}$ & $\begin{array}{c}0.67 \\
(.501)\end{array}$ & $\begin{array}{l}5.97 \pm 1.30 \\
5.76 \pm 1.35\end{array}$ & $\begin{array}{c}0.42 \\
(.673)\end{array}$ \\
\hline $\begin{array}{l}\text { Have you vaccinated } \\
\text { your children for } \\
\text { hepatitis A? }\end{array}$ & $\begin{array}{l}\mathrm{No}^{\mathrm{a}} \\
\mathrm{Yes}^{\mathrm{b}} \\
\text { Do not know }\end{array}$ & $\begin{array}{l}27(27.0) \\
59(59.0) \\
14(14.0)\end{array}$ & $\begin{array}{l}5.35 \pm 1.49 \\
6.59 \pm 0.65 \\
6.16 \pm 1.42\end{array}$ & $\begin{array}{c}12.47 \\
(<.001) \\
a<b^{*}\end{array}$ & $\begin{array}{l}5.60 \pm 0.88 \\
5.88 \pm 0.70 \\
5.35 \pm 0.97\end{array}$ & $\begin{array}{c}2.95 \\
(.057)\end{array}$ & $\begin{array}{l}4.98 \pm 1.17 \\
5.89 \pm 1.12 \\
5.09 \pm 1.65\end{array}$ & $\begin{array}{l}6.21 \\
(.003) \\
a<b^{*}\end{array}$ & $\begin{array}{l}4.97 \pm 1.49 \\
6.41 \pm 0.81 \\
5.97 \pm 1.57\end{array}$ & $\begin{array}{c}14.37 \\
(<.001) \\
a, c<b^{*}\end{array}$ \\
\hline $\begin{array}{l}\text { Do you plan to vaccinate } \\
\text { your children for } \\
\text { hepatitis A? }\end{array}$ & $\begin{array}{l}\text { No } \\
\text { Yes }\end{array}$ & $\begin{array}{l}16(16.0) \\
84(84.0)\end{array}$ & $\begin{array}{l}4.47 \pm 1.49 \\
6.53 \pm 0.73\end{array}$ & $\begin{array}{c}8.56 \\
(<.001)\end{array}$ & $\begin{array}{l}5.36 \pm 0.95 \\
5.80 \pm 0.77\end{array}$ & $\begin{array}{c}2.01 \\
(.047)\end{array}$ & $\begin{array}{l}4.52 \pm 1.26 \\
5.73 \pm 1.20\end{array}$ & $\begin{array}{c}3.67 \\
(<.001)\end{array}$ & $\begin{array}{l}4.02 \pm 1.41 \\
6.33 \pm 0.88\end{array}$ & $\begin{array}{c}8.59 \\
(<.001)\end{array}$ \\
\hline
\end{tabular}

*Scheffé test, $p<.050$.

Table 3. Correlations among Attitude toward Hepatitis A Vaccination, Perceived Behavioral Control, Subjective Norm, and Vaccination Intention

\begin{tabular}{|c|c|c|c|c|}
\hline \multirow[t]{2}{*}{ Variables } & Attitude & $\begin{array}{c}\text { Perceived behavioral } \\
\text { control }\end{array}$ & Subjective norm & Intention \\
\hline & $\mathrm{r}(p)$ & $\mathrm{r}(p)$ & $\mathrm{r}(p)$ & $\mathrm{r}(p)$ \\
\hline Attitude & 1 & & & \\
\hline Perceived behavioral control & $.57(<.001)$ & 1 & & \\
\hline Subjective norm & $.53(<.001)$ & $.45(<.001)$ & 1 & \\
\hline Intention & $.91(<.001)$ & $.58(<.001)$ & $.56(<.001)$ & 1 \\
\hline
\end{tabular}

tude [24], it is meaningful that this study investigated mothers' attitudes and health beliefs regarding vaccination.

In the present study, mothers' attitudes toward HA vaccination were relatively positive. Participants also reported rel- atively high scores for perceived behavioral control, subjective norm, and vaccination intention. In other words, Korean mothers' attitudes toward HA vaccination were relatively positive. In addition, they felt that vaccination was straightfor- 
Table 4. Factors Influencing Hepatitis A Vaccination Intention

\begin{tabular}{|c|c|c|c|c|c|}
\hline Factor & $\mathrm{B}$ & SE & $\beta$ & $\mathrm{t}$ & $p$ \\
\hline (Constant) & -1.38 & 0.89 & & 0.88 & .379 \\
\hline Attitude toward hepatitis A vaccination & 0.94 & 0.05 & .85 & 17.81 & $<.001$ \\
\hline Norm of hepatitis A vaccination & 0.10 & 0.05 & .10 & 2.17 & .032 \\
\hline
\end{tabular}

ward to accomplish, and the social pressure for HA vaccination was relatively high. Yoon et al. [24] reported that the HA vaccination rate for parents was $2.5 \%$, and that for their youth was $0.6 \%$. However, in the present study, the HA vaccination rate for mothers was 35\%, and their children's rate was $59 \%$. These results show that parents who were vaccinated against HA had a more positive attitude and higher HA vaccination-intention scores. The HA vaccination rate for children in the present study was similar to the result reported in the study conducted by Yoon et al. [8] of participants under the age of 10 living in central Korea, but it was higher than the rate of $42.2 \%$ of kindergarten children in the United States reported by Bardenheier et al. [11]. Although studies have shown that low socioeconomic status was related to a low vaccination rate, Bardenheier et al. [11] reported that mothers who had 12 or fewer years of education and had an annual income under US\$50,000 demonstrated a higher likelihood of having received an HA vaccination. However, no significant difference emerged according to income in the present study. In addition, no difference in attitude toward $H A$ vaccination, perceived behavioral control, subjective norm, and vaccination intention emerged between mothers who had graduated from high school and those with university degrees.

In the present study, many participants planned to vaccinate their children against HA. However, the actual HA vaccination rate was 59\%, which is somewhat lower than the rate for intention. Yoon et al. [24] found that the main reason participants reported not receiving vaccination against HA was that they were unaware of the need for HA vaccination or its vaccination schedule. Bardenheier et al. [11] reported that influencing factors against HA vaccination included the lack of a doctor's or health department's recommendation and the lack of parental awareness. Therefore, this suggests that vaccination education, including the vaccination schedule, is essential for increasing HA vaccination rates.

In the study of Bardenheier et al. [11], a significant difference in attitude towards HA vaccination depending on awareness of HA was found, but no significant difference emerged in the present study. In Korea, vaccination against HA was not mandatory until April 2015. Vaccination of children under 19 was therefore performed when parents chose that option.
Thus, parents' perceptions and attitudes had an effect on children's vaccination [24]. In the present study, participants had a relatively high education level, so they seemed to be aware of the necessity of HA vaccination.

In the present study, the strongest influencing factor of intention to give children the HA vaccination was mothers' attitude toward HA vaccination, followed by subjective norm. The study results suggest that higher vaccination intention is present when mothers have a positive attitude toward HA vaccination and when they experience increased pressure to vaccinate from others. Kim and Choi [27], who conducted research on mothers' intention of HPV vaccination based on the $\mathrm{TPB}$, also showed a similar result to that of the current study: mothers' attitude and subjective norms were significant predictors for intention of HPV vaccination. Park [28] reported that the more positive attitude on preventive health behavior of hepatitis, the higher the compliance with preventive behaviors. This result is similar to the results of the present study. In addition, Park [28] stated that it is likely for Koreans to be infected by HA over time, because Korean culture includes passing glasses around or sharing food in a bowl, providing many opportunities for infection in group situations such as military service and overseas travel, which increases every year. The Korea Centers for Disease Control and Prevention [1] stated that prevention is most crucial for HA, suggesting vaccination, hand washing, boiling water, eating cooked food, and hygienic cooking as prevention methods. Therefore, it is necessary to promote the effectiveness and benefits of HA vaccination, to provide free infant immunization, to promote personal hygiene.

This study was limited in that it only included participants from Seoul and Gyeonggi Province in Korea. Therefore, it would be difficult to generalize the study results to all mothers with children and adolescents. Follow-up studies on HA vaccination knowledge, intention, and health beliefs of mothers from various backgrounds are therefore proposed.

\section{CONCLUSION}

This study showed that the attitude of mothers with children under 19 toward HA vaccination was very positive, and 
their scores for perceived behavioral control, subjective norm, and vaccination intention were relatively high. Attitude toward HA vaccination was the strongest influencing factor, followed by subjective norm. Therefore, providing information on the importance of HA vaccination and the vaccination schedule would help develop positive attitude toward vaccination. In addition, efforts to enhance the subjective norm of HA vaccination are also needed to improve perceptions of the importance of HA vaccination. These efforts should include public service announcements and educational programs offered at the institutional (schools and medical establishments) and governmental levels.

\section{Conflict of interest}

No existing or potential conflict of interest relevant to this article was reported.

\section{REFERENCES}

1. Korea Centers for Disease Control and Prevention. Disease information: Hepatitis A [internet]. Cheongju: Korea Centers for Disease Control and Prevention; 2012 [cited 2018 January 2]. Available from:

http://www.cdc.go.kr/CDC/health/CdcKrHealth0101.jsp?menu Ids=HOME001-MNU1132-MNU1147-MNU0746-MNU2422\&fid= $7955 \&$ cid $=77617$.

2. Korean Society for Healthcare-associated Infection Control and Prevention. Infection control and prevention in healthcare facilities. 5th ed. Seoul: Hanmi pub; 2017. p. 647-668.

3. Kim JM, Lee YS, Lee JH, Kim W, Lim KS. Clinical outcomes and predictive factors of spontaneous survival in patients with fulminant hepatitis A. The Korean Journal of Hepatology. 2008;14(4): 474-482. https://doi.org/10.3350/kjhep.2008.14.4.474

4. Vacchio MN. Incidence of hepatitis A in Argentina after vaccination. Journal of Viral Hepatitis. 2008;15(s2):47-50. https://doi.org/10.1111/j.1365-2893.2008.01029.x

5. Song YB, Lee JH, Choi MS, Koh KC, Paik SW, Yoo BC, et al. The age-specific seroprevalence of hepatitis A virus antibody in Korea. The Korean Journal of Hepatology. 2007;13(1):27-33.

6. Kim CY, Hong WS. Seroepilemiology of type A and type B hepatitis in Seoul area. The Korean Journal of Medicine. 1982;25(1):19-27.

7. Lee H, Cho HK, Kim JH, Kim KH. Seroepidemiology of hepatitis A in Korea: Changes over the past 30 years. Journal of Korean Medical Science. 2011;26(6):791-796.

https://doi.org/10.3346/jkms.2011.26.6.791

8. Yoon SW, Lee WK, Cho SY, Moon SH, Shin HD, Yun SY, et al. The seroprevalence rate, vaccination rate and seroconversion rate of hepatitis A in central region of Korea. Korean Journal of Gastroenterology. 2011;57(3):166-172.
9. Oh HY, Park JY. Immunization, knowledge, and preventive health behaviors to hepatitis A in university students. Korean Journal of Health Education and Promotion. 2011;28(5):83-95.

10. Choi KA, Kim JH, Lee KS, Oh JK, Liu SN, Shin HR. Knowledge of human papillomavirus infection and acceptability of vaccination among adult women in Korea. Korean Journal of Obstetrics and Gynecology. 2008;51(6):617-623.

11. Bardenheier B, González IM, Washington ML, Bell BP, Averhoff F, Massoudi MS, et al. Parental knowledge, attitudes, and practices associated with not receiving hepatitis A vaccine in a demonstration project in Butte County, California. Pediatrics. 2003;112(4): e269. https://doi.org/10.1542/peds.112.4.e269

12. Ajzen I. From intentions to actions: A theory of planned behavior. In: Kuhl J, Beckmann J, editors. Action Control. SSSP Springer Series in Social Psychology. Berlin: Springer; 1985. p. 11-39. https://doi.org/10.1007/978-3-642-69746-3_2

13. Lee JK, Kim HE. A study on the effects of television viewing on purchase intention for women of environmentally friendly cosmetic products: Focusing on the viewing hours of TV program genres, perception of concern about skin health, and the variables of the theory of planned behavior. Journal of Communication Science. 2010;10(3):463-497.

14. Park HR. Internet purchase behavior based on anticipated emotion induced theory of planned behavior: Focused on gender difference. The Korean Journal of Consumer and Advertising Psychology. 2010;11(4):661-686.

https://doi.org/10.21074/kjlcap.2010.11.4.661

15. Hur T, Han M, Kim Y. Gender differences of risk-taking decisions in sexual behaviors: Condom use and theory of planned behavior. The Korean Journal of Woman Psychology. 2004;9(3):69-87.

16. Kim HS, Nam ES. Prediction of breastfeeding intentions and behavior: An application of the theory of planned behavior. The Journal of Nurses Academic Society. 1997;27(4):796-806. https://doi.org/10.4040/jnas.1997.27.4.796

17. Armitage CJ, Conner M. Efficacy of the theory of planned behaviour: A meta-analytic review. British Journal of Social Psychology. 2001;40(4):471-499. https://doi.org/10.1348/014466601164939

18. Ogilvie GS, Remple VP, Marra F, McNeil SA, Naus M, Pielak KL, et al. Parental intention to have daughters receive the human papillomavirus vaccine. Canadian Medical Association Journal. 2007; 177(12):1506-1512. https://doi.org/10.1503/cmaj.071022

19. Askelson NM, Campo S, Lowe JB, Smith S, Dennis LK, Andsager J. Using the theory of planned behavior to predict mothers' intentions to vaccinate their daughters against HPV. The Journal of School Nursing. 2010;26(3):194-202. https://doi.org/10.1177/1059840510366022

20. Juraskova I, O'Brien M, Mullan B, Bari R, Laidsaar-Powell R, McCaffery K. HPV vaccination and the effect of information framing on intentions and behaviour: An application of the theory of planned behaviour and moral norm. International Journal of Behavio- 
ral Medicine. 2012;19(4):518-525.

https://doi.org/10.1007/s12529-011-9182-5

21. Baek JS, Han MA, Park J, Yun NR. Hepatitis A virus vaccination status and related factors among college students. Korean Journal of Health Promotion. 2014;14(3):103-111.

https://doi.org/10.15384/kjhp.2014.14.3.103

22. Kim KJ, Hwang T, Lee KS. Knowledge, health belief, and vaccination behavior on hepatitis A among university students. Journal of Agricultural Medicine and Community Health. 2016;41(3):119-128.

23. Ajzen I. The theory of planned behavior. Organizational Behavior and Human Decision Processes. 1991;50(2):179-211. https://doi.org/10.1016/0749-5978(91)90020-T

24. Yoon SH, Lee HY, Kim HW, Kong KA, Kim KH. Adolescents' and parental knowledge, health beliefs toward hepatitis A vaccination. Korean Journal of Pediatric Infectious Diseases. 2013;20(3):147-160.

25. Kim ES, Yoo JH, Hong JI. Introduction of hepatitis A vaccination into the national immunization program in Korea. Public Health Weekly Report. 2015;8(32):759-760.

26. Kim SM. Nine out of ten people in their 20s do not have hepatitis A antibody. The Chosun Ilbo [Internet]. 2017 December 14 [cited 2018 April 5]: Society. Available from:

http://news.chosun.com/site/data/html_dir/2017/12/14/2017 121400324.html.

27. Kim KM, Choi JS. Mothers' intentions to vaccinate their teenaged children against human papillomavirus, as predicted by sex in South Korea: An application of the theory of planned behavior. Japan Journal of Nursing Science. 2017;14(4):288-296. https://doi.org/10.1111/jjns.12155

28. Park JY. Preventive behavior and health belief about hepatitis A of adults in their twenties to thirties. Korean Journal of Adult Nursing. 2011;23(4):403-411. 